\title{
Ideal barriers to polarization reversal and domain-wall motion in strained ferroelectric thin films
}

\author{
S. P. Beckman $*$ Xinjie Wang, Karin M. Rabe, and David Vanderbilt \\ Department of Physics and Astronomy, Rutgers University, Piscataway, New Jersey 08854-8019
}

(Dated: May 30, 2018)

\begin{abstract}
The ideal intrinsic barriers to domain switching in $c$-phase $\mathrm{PbTiO}_{3}(\mathrm{PTO}), \mathrm{PbZrO}_{3}(\mathrm{PZO})$, and $\mathrm{PbZr}_{1-x} \mathrm{Ti}_{x} \mathrm{O}_{3}(\mathrm{PZT})$ are investigated via first-principles computational methods. The effects of epitaxial strain on the atomic structure, ferroelectric response, barrier to coherent domain reversal, domain-wall energy, and barrier to domain-wall translation are studied. It is found that PTO has a larger polarization, but smaller energy barrier to domain reversal, than PZO. Consequentially the idealized coercive field is over two times smaller in PTO than PZO. The Ti-O bond length is more sensitive to strain than the other bonds in the crystals. This results in the polarization and domain-wall energy in PTO having greater sensitivity to strain than in PZO. Two ordered phases of PZT are considered, the rock-salt structure and a (100) PTO/PZO superlattice. In these simple structures we find that the ferroelectric properties do not obey Vergard's law, but instead can be approximated as an average over individual 5-atom unit cells.
\end{abstract}

PACS numbers: 77.80.Fm 77.84.Dy 77.55.+f

\section{INTRODUCTION}

The advent of advanced techniques for the growth of high-quality ferroelectric thin-films ${ }^{1}$ has spawned a great deal of interest in using these films for high-density nonvolatile ferroelectric information storage $\stackrel{2,3,4}{2}$ The device concept involves associating 0 and 1 bits with "up" and "down" polarized domains in the film,$\frac{4,5}{}$

For this application, assuring the retention of the data is of critical importance $\stackrel{\underline{4}}{\underline{4}}$ It is necessary therefore to establish an understanding of the factors that govern the stability of polarization domains. As will be seen from the discussion below, the domain-wall energy and mobility are especially important. One promising avenue to control these critical factors is by tuning the epitaxial strain in the thin films, present due to lattice mismatch between the film material and the substrate. Both the investigation of the stability mechanisms and of the effects of epitaxial strain can be carried out using first principles methods, as will be described further below.

Polarization switching is believed to occur via the nucleation of an oppositely polarized domain at a defect such as a dislocation or inclusion, followed by domain wall advancement outward from the embryonic domain. The wall does not propagate uniformly, but by a mechanism of kink nucleation and motion initiated at a defect or compositional inhomogeneity where the local barrier is reduced. Subsequently this small region expands lateral to the original wall, converting the phase as it passes $\frac{6}{6}$ This process is complicated and depends strongly on extrinsic effects such as the quality of the film, the processing conditions, the mechanical, thermal, and electrical histories, as well as the environmental conditions at the surface of the film.

Underpinning the extrinsic behavior is the intrinsic, atomic nature of the ferroelectric material. We focus here on two levels of idealized intrinsic behavior. First, we consider the barrier for coherent domain reversal, in which every unit cell in the crystal undergoes simultaneous reversal from one ferroelectric ground state to its opposite one. This is a purely bulk property, since the crystal is assumed to maintain its three-dimensional periodicity throughout, and the barrier is determined by a saddle point corresponding to the centrosymmetric (paraelectric) bulk structure.

Second, we consider the idealized barrier for a domain wall to translate by one unit cell in the direction normal to the wall, while always maintaining two-dimensional periodicity in the parallel directions. This ideal barrier for domain-wall motion is determined by a saddlepoint structure in which the domain wall is centered at an unfavorable position in the unit cell. In both cases, we focus on zero-temperature transition paths. Firstprinciples calculations have been performed to examine $180^{\circ}$ domain walls in $\mathrm{PbTiO}_{3}, \frac{7}{,}$ and valence-bond methods have been employed to study domain-wall motion in $\mathrm{PbZr}_{1-x} \mathrm{Ti}_{x} \mathrm{O}_{3} \underline{\underline{6}}$

The energy to reverse the polarization via the coherentreversal or domain-wall-translation path is proportional to the volume of the film or to the area of the domain wall respectively, and thus may be orders of magnitude larger than the true thermal barrier for motion of the domain wall. However, even if it is not quantitatively relevant to the real mechanisms of domain-wall motion, the study of the idealized barriers give insight into the underlying atomistic mechanisms and materials properties that are relevant to real domain-wall motion. Thus, any more sophisticated treatments that may be put forward in the future should start from a firm understanding of these intrinsic effects.

The impact of strain on a crystal's ferroelectric response has been theoretically studied using both phenomenological and first principles techniques. For singlephase compounds, epitaxial strain phase diagrams have been obtained both from the Landau-Devonshire freeenergy ${ }^{\underline{8}}$ and from first-principles effective-Hamiltonian 
methods $\stackrel{9,10}{10}$ The effect of strain has also been studied in superlattices $\stackrel{11}{\underline{11}}$ However the effect of composition and strain on polarization reversal and domain-wall energy has yet to be reported in detail.

In this paper, the ideal barriers to coherent polarization reversal and domain-wall motion are studied for c-phase $\mathrm{PbTiO}_{3}$ (PTO), $\mathrm{PbZrO}_{3}(\mathrm{PZO})$, and two ordered forms of $\mathrm{PbZr}_{1-x} \mathrm{Ti}_{x} \mathrm{O}_{3}$ (PZT), the rock-salt structure and a (100)-oriented superlattice. In particular, we report the dependence of the structure, the spontaneous ferroelectric polarization, the barrier to coherent domain reversal, the domain-wall energy, and the barrier to domain-wall translation as a function of epitaxial strain.

The manuscript is organized as follows. In Sec. I we discuss the boundary conditions used in our approach, present the geometries of the cells used to treat pure PTO and PZO and the ordered PZT alloys, and give the details of the computational methods that were used. The results of the calculations are presented in Sec. III. A more detailed analysis of the crystal structures and an identification of the relevant atomistic features is given in Sec.IV. Finally, we summarize and conclude in Sec.V

\section{THEORETICAL APPROACH}

\section{A. Boundary conditions}

In the study of ferroelectric thin films, one has to specify both the mechanical boundary conditions, such as epitaxial strain constraints,,$\underline{\underline{9}}$ as well as the electrical boundary conditions. In the present calculations, periodic boundary conditions are used. This corresponds to zero macroscopic field such as would be obtained with ideal symmetric short-circuited electrodes. Thus, these results may not apply in film geometries in which an electrical bias is intentionally or unintentionally present, $\underline{12,13,14}$ We further note that these periodic boundary conditions produce "constrained bulk" calculations, similar to those of Ref. [9], in which we carry out a bulk calculation on a primitive unit cell whose basal lattice vectors are constrained to form a simple square lattice having a specified lattice parameter $a$. This allows us to isolate the effects of epitaxial strain from surface and interface effects which would also be present in a real film.

\section{B. Structures: PTO and PZO}

For ferroelectric PTO and PZO we assume tetragonal P4mm symmetry with 5 atoms per unit cell (point group $\mathrm{C}_{4 v}$ ). We take the normal to the film to be the 4-fold axis along $z$. In the paraelectric case, there is an additional mirror symmetry perpendicular to z, resulting in point group $\mathrm{D}_{4 h}$. The degrees of freedom that are relaxed to obtain the lowest-energy configuration are the magnitude $c$ of the lattice vector along $z$ and the atomic displacements, all along $\mathrm{z}$, that are consistent with the symmetry constraints.. The calculations are carried out as a function of the epitaxial strain, i.e., in-plane lattice constant $a$. Restricting the calculations to a single tetragonal phase allows for an examination of the intrinsic properties of the crystal in a continuous manner, without considering abrupt changes due to possible strain-induced phase transitions.

Domain-wall energies and the barriers to domain-wall motion are studied using the methods outlined in Meyer and Vanderbilt. $\underline{7}$ For pure PTO and PZO, the domain wall is modeled using an $N \times 1 \times 1$ supercell containing $N / 2$ "up" unit cells and $N / 2$ "down" cells. It is found that the domain walls are sufficiently thin that the energies are converged for $N=6$; however, supercells of size $N=8$ are used to investigate the local structure. Epitaxial constraints are applied to the domain-wall calculations, as follows. The $c$ parameter is held fixed at the computed value for the bulk ferroelectric structure, appropriate for an isolated domain wall far from any surfaces or interfaces, and the in-plane lattice parameters are fixed at $N a$ and $a$, which define the strain state of the system. The symmetries of the supercell are selected such that the domain wall is centered either on a $\mathrm{PbO}$ plane or on a $\mathrm{TiO}_{2}$ (or $\mathrm{ZrO}_{2}$ ) plane. In the simplest scenario, the difference of the energies of these two configurations would give the idealized barrier for domain-wall motion.

\section{Structures: PZT}

Random alloys such as PZT $\left(\mathrm{PbZr}_{1-x} \mathrm{Ti}_{x} \mathrm{O}_{3}\right)$ pose a challenge to first-principles methods because the atomic arrangement is not naturally compatible with periodic boundary conditions. One approach to addressing this problem is to construct large supercells in which the cation arrangement approaches randomness, either by randomizing the occupation of each cation site in the supercell or by the application of techniques for constructing so-called special quasirandom structures $\underline{15}$ Using effective-Hamiltonian methods or parameterized interatomic potentials, it may be possible go to quite large supercells,$\frac{16,17}{1}$ but one is typically limited to rather small supercells when using direct first-principles methods.

To study PZT at $x=0.50$, we here adopt this supercell approach, considering two minimal supercells: (i) a superlattice structure with alternating layers of PTO and PZO in the (100) direction, and (ii) a rock-salt structure (or "3D chess board") in which the chemical identity alternates between all nearest $\mathrm{B}-\mathrm{B}$ neighbors in the $\mathrm{ABO}_{3}$ structure. We again constrain the in-plane lattice constants to equal those of an assumed cubic substrate, and impose orthorhombic $\mathrm{C}_{2 v}$ or tetragonal $\mathrm{C}_{4 v}$ symmetry for the (100)- and rock-salt-ordered superlattices respectively. In the paraelectric case, when the polarization vanishes, the corresponding symmetry labels are 
$\mathrm{D}_{2 h}$ and $\mathrm{D}_{4 h}$

\section{Computational Methods}

Density-functional theory calculations are performed using the local-density approximation $\frac{18,19}{19}$ as implemented in the PWscf code package ${ }^{20}$ Ultrasoft pseudopotentials 21,22 are used in place of the all-electron ion potentials. The Kohn-Sham wave functions are expressed in terms of a plane-wave expansion, and the Brillouin zone is sampled using the Monkhorst-Pack algorithm. ${ }^{23}$ The coherent reversal calculations were performed with a 35 Ry plane-wave cutoff and $6 \times 6 \times 6$ k-point sampling, while the domain-wall calculations were carried out using a 30 Ry cutoff and $4 \times 4 \times 2$ sampling; in all cases these were chose to ensure that the forces are converged to better than $15 \mathrm{meV} / \AA$. The Berry-phase technique $\underline{10}$ is used to calculate the polarization of the structures.

With these choices the lattice parameters of known structures are calculated to be near experimental values for PTO, $a_{0}^{\exp }=3.90 \AA$ and $c_{0}^{\exp }=4.15 \AA \stackrel{24,25}{2 n}$ particular, we obtain equilibrium lattice parameters for the tetragonal ferroelectric phases of PTO and $\mathrm{PZO}$ of $a_{0}^{\mathrm{PTO}}=3.86 \AA, c_{0}^{\mathrm{PTO}}=4.02 \AA, a_{0}^{\mathrm{PZO}}=4.09 \AA$, and $c_{0}^{\mathrm{PZO}}=4.16 \AA$. The calculated lattice parameters for the PZT structures (with symmetry imposed as explained above) are $a_{0}^{\mathrm{PZT}}=3.97 \AA$ and $c_{0}^{\mathrm{PZT}}=4.09 \AA$, consistent with the predictions of Vegard's law. All epitaxial strain values reported in this paper are defined relative to the corresponding ground-state $a_{0}$ values given here.

\section{RESULTS}

\section{A. Structural properties of paraelectric and ferroelectric states}

Figure 1 shows a sketch of the double-well potential of a typical ferroelectric, indicating the structure of one of the local ferroelectric minima and of the paraelectric saddle-point configuration. We calculate the energy, $c / a$ ratio, and polarization for these ferroelectric and paraelectric states in PTO, PZO, and PZT by relaxing $c$ as well as all internal coordinates subject to the appropriate symmetries specified in the previous section.

In Fig. 2 we present our computed values of the $c / a$ ratio as a function of epitaxial strain for both symmetries. Applying a compressive in-plane strain causes $c$ (and $c / a$ ) to increase, which corresponds to the common situation where the Poisson ratio is positive. This increase is roughly linear and composition-independent in the paraelectric case, shown in Fig. 2(a), where the polarization plays no role. However, in the ferroelectric case, the curves are steeper, more non-linear, and more composition-dependent, as shown in Fig. 2(b), where the electric polarization is enhanced by compressive in-plane strain.

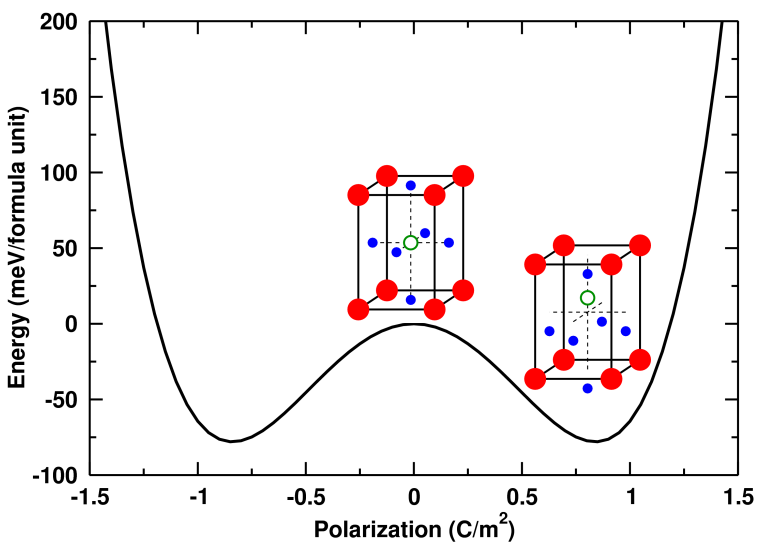

FIG. 1: Double-well potential of PTO at zero in-plane strain. The ferroelectric state (minimum sketched at right) has pointgroup symmetry $\mathrm{C}_{4 v}$; the paraelectric state (saddle point sketched at center) has $\mathrm{D}_{4 h}$.

The enhancement of polarization by compressive epitaxial strain can be seen clearly in Fig. 31(a). The polarization is generally largest in PTO, smallest in PZO, and intermediate in PZT. This ordering is consistent with the variation in the $c / a$ ratios presented in Fig. 2. However, Fig. 3(b) shows that the height of the barrier is actually larger for PZO than for PTO, with the PZT alloys again lying at intermediate values.

\section{B. Double-well potential and domain reversal}

A double well potential as a function of polarization is obtained as a fourth order polynomial

$$
E(P)=a P^{2}+b P^{4},
$$
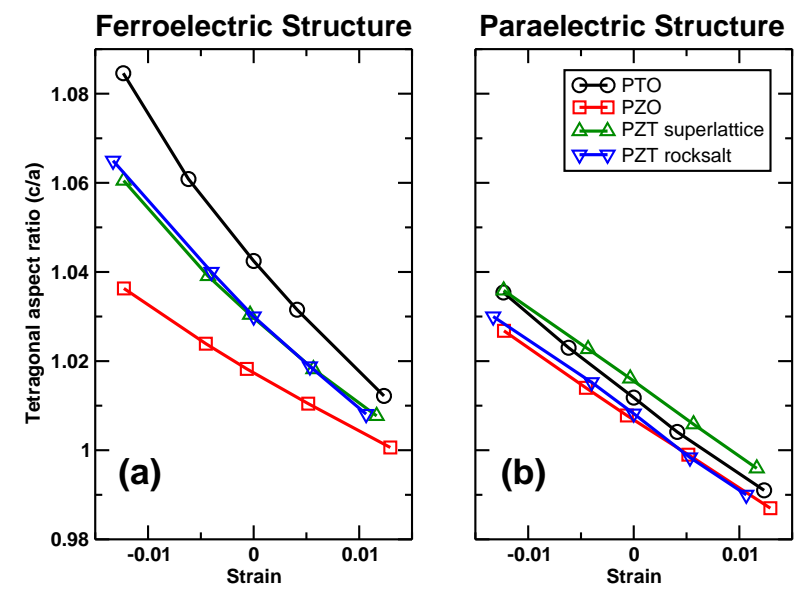

FIG. 2: Lattice-constant ratio $c / a$ versus applied epitaxial strain, obtained by relaxing in the ferroelectric (a) or paraelectric (b) state. 

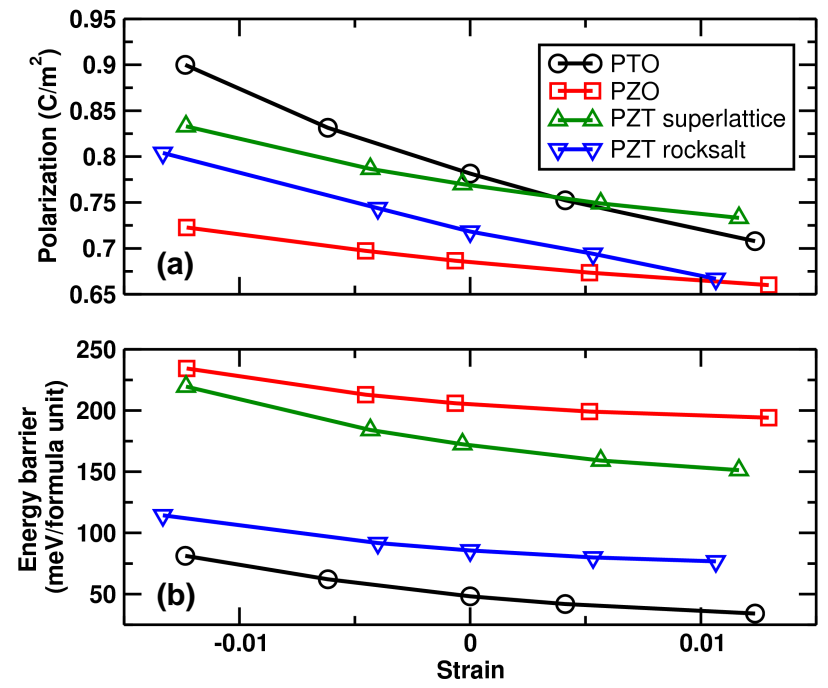

FIG. 3: Polarization (a) and energy-barrier height (b) versus applied epitaxial strain in tetragonal PTO-PZO systems.

where $E$ is the energy (relative to the paraelectric saddle point) and $P$ is the polarization. Knowing the values $P_{1}$ and $E_{1}$ that characterize the ferroelectric minimum, Eq. (1) can be rearranged to find $a=2 E_{1} / P_{1}^{2}$ and $b=-E_{1} / P_{1}^{4}$. Figure 1 shows the double-well potential of ferroelectric PTO at zero in-plane strain. The various double-well potentials can conveniently be analyzed by fitting them to this simplified functional form. ${ }^{26}$ For example, the shapes of the double-well potentials are seen to be rather different for PTO and PZO, with the latter having a deeper minimum that however occurs at a smaller value of polarization.

Introducing the electric enthalpy $F=E-\mathcal{E} P$, where $\mathcal{E}$ is the electric field, and recalling that $\mathcal{E}=d E / d P$ corresponds to the slope of the $E(P)$ curve, we obtain

$$
\mathcal{E}_{\mathrm{c}}=(4 / 3)^{3 / 2} E_{1} / P_{1}
$$

for the ideal intrinsic coercive field, defined as the maximum slope attained between the minimum and saddle point. It should be emphasized that this is an artificial quantity that may be orders of magnitude larger than physical coercive fields; it corresponds to the field at which the minority domain ceases to be defined theoretically, whereas physical coercive fields are defined by the field at which domain walls become unpinned. In comparison, Lee et al. find coercive fields of $\sim 25 \mathrm{MV} / \mathrm{m}$ in $\mathrm{PbZr}_{0.2} \mathrm{Ti}_{0.8} \mathrm{O}_{3}$ thin films of $100 \mathrm{~nm}$ thickness.

Using Eqs. (1,2) and the data in Fig. 3, the coercive field is calculated as a function of applied strain and plotted in Fig. 4. The ideal coercive field can be seen to be much larger for PZO than for PTO (with PZT at intermediate values). This is consistent with the trends identified earlier, since $E_{1}$ increases while $P_{1}$ decreases in going from PTO to PZO, and from Eq. (2) it is obvious that both of these effects tend to increase the ideal

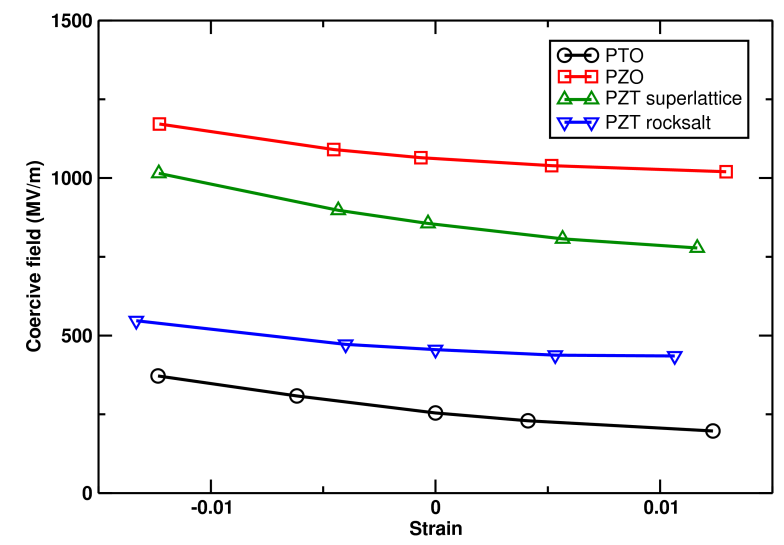

FIG. 4: Ideal coercive field versus epitaxial strain as determined from Eq. (2) using the data from Fig. 3 .

coercive field. The observed strain dependence of the polarization and coercive field indicate that the application of compressive strain expands the hysteresis loops and applying a tensile strain contracts them.

From the simple model of Eq. (1) it is also straightforward to plot the polarization as a function of electric field in order to obtain ideal hysteresis curves such as those plotted in Fig. 5] Again we emphasize that this corresponds to coherent domain reversal, i.e., a scenario in which the reversal happens simultaneously everywhere without loss of crystal periodicity. The ideal coercive field can be seen to be much larger for PZO than for PTO, consistent with Fig. 4.

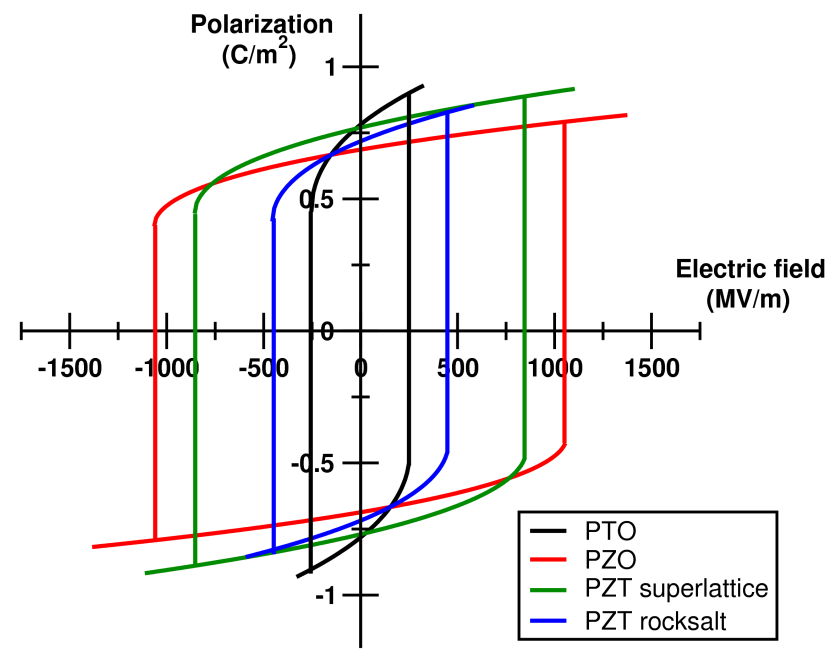

FIG. 5: Hysteresis curves calculated from Eq. (11) at zero epitaxial strain. 


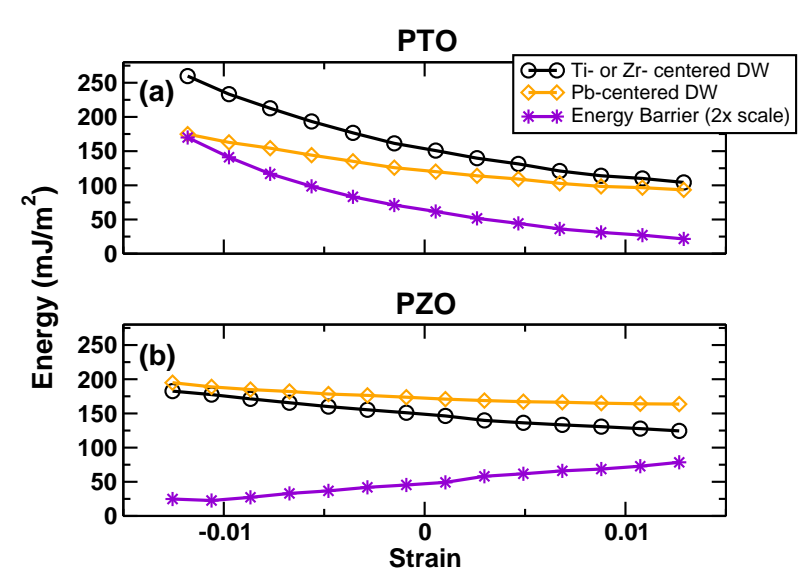

FIG. 6: Domain wall energy and barrier to domain-wall motion in strained PTO (a) and PZO (b) films. The energy barrier is scaled $2 \times$.

\section{Domain walls}

The energies of domain walls are calculated for PTO, PZO, and the (100) superlattice PZT. For all three crystals the supercell belongs to the $\mathrm{D}_{2 h}$ point group. We constrain the domain-wall location by enforcing an inversion symmetry about a center in the specified plane, and relax all other internal degrees of freedom. For PTO and $\mathrm{PZO}$ the domain-wall energies are shown on the right axis of Fig. 6. These increase with compressive strain, for which the polarization is larger and therefore the cost of introducing an unpolarized layer is larger. A simple estimate of the energy barrier to domain-wall motion is the difference between the two domain-wall energies, which is plotted along the left axis of Fig. 6. These barriers have a strain dependence quite different in PTO than in PZO. We shall discuss this behavior further in Sec. IVB,

The domain-wall energies are shown for the (100) PZT superlattice in the top frame of Fig. 7(a). Here it is possible to specify a domain wall located on a $\mathrm{ZrO}_{2}$ or $\mathrm{TiO}_{2}$ plane, but not on a $\mathrm{PbO}$ plane, because the compositional order makes it impossible to choose an inversion center in a $\mathrm{PbO}$ plane. As is evident in the figure, the Zr-centered domain wall is lower in energy at all strains. If we choose initial atomic coordinates corresponding to a domain wall that is positioned on a $\mathrm{PbO}$ plane, we find that the atomic relaxations spontaneously carry the domain wall toward the nearest $\mathrm{ZrO}_{2}$ plane, consistent with the fact that it is not pinned by symmetry constraints. Figure 7 (c) shows a sketch of the resulting energy landscape for domain-wall motion in this superlattice system. We have not attempted to calculate (or even to precisely define) the energy surface between the $\mathrm{TiO}_{2}$ and $\mathrm{ZrO}_{2}$ planes, so the dashed lines in the Fig. 7(c) are just conjectural.
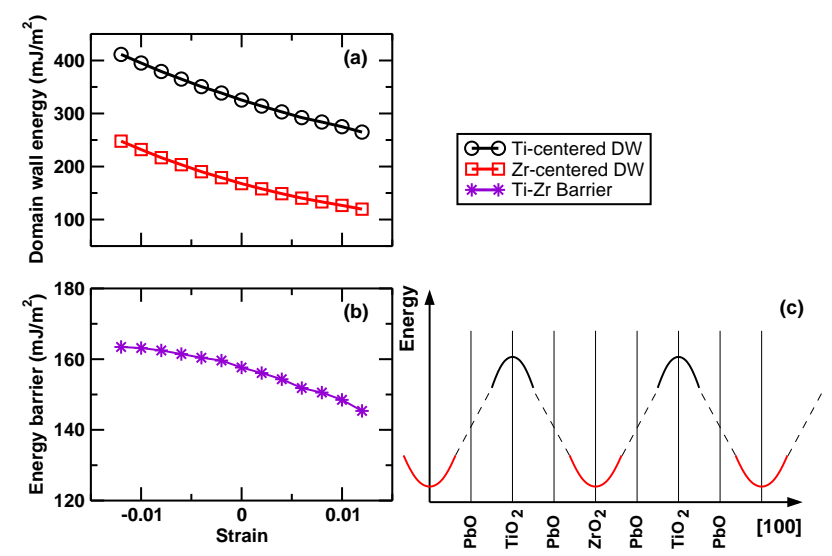

FIG. 7: Domain-wall energy (a) and barrier to domain-wall motion (b) in a strained (100) PZT superlattice. Sketch of energy landscape emerging from the calculations (c).

\section{DISCUSSION}

In this section we take a closer look at the results presented in Sec. III seeking to explain some of those results in terms of interactions at the atomistic level. The influence of local strain on the structure will be examined.

\section{A. Polarization and polarization reversal \\ 1. Dependence of polarization on composition}

In Fig. 2(a) it can be seen that the $c / a$ ratio for ferroelectric PZT obeys Vegard's law quite well, being very close to the average of PTO and PZO at all strains. However, this is not the case for the polarization in Fig. 3(a), where the PZT superlattice and rock-salt structures lie above and below the average, respectively.

This can be understood by inspecting the local strains and how they impact the properties of the crystal. Looking first at the superlattice case, while the periodicity is $2 a_{0}^{\mathrm{PZT}}=7.94 \AA$ in the (100) direction, the $\mathrm{Pb}$ atoms are not uniformly spaced. The spacing is 3.81 and $4.14 \AA$ across the $\mathrm{TiO}_{2}$ and $\mathrm{ZrO}_{2}$ planes respectively. Defining the local strain in the (100) direction relative to the equilibrium lattice parameters $\left(a_{0}^{\mathrm{PTO}}=3.86\right.$ and $\left.a_{0}^{\mathrm{PZO}}=4.09 \AA\right)$, the PTO layer has a strain of $-1.3 \%$ while that of the $\mathrm{PZO}$ layer is $+1.2 \%$. Using the data for strained PTO and PZO in Fig. 3(a) and applying these local strains, we can estimate the polarization contribution to be 0.91 and $0.66 \mathrm{C} / \mathrm{m}^{2}$ from the PTO and PZO layers respectively. The average of these is $0.79 \mathrm{C} / \mathrm{m}^{2}$, which is very close to the calculated Berry-phase value of $0.77 \mathrm{C} / \mathrm{m}^{2}$. A similar analysis applies to the rock-salt case. The $\mathrm{Pb}$ separation in both the (100) and (010) directions is $3.97 \AA$, corresponding to a strain of $\pm 2.8 \%$ in the PTO and PZO cells respectively. Extrapolating 


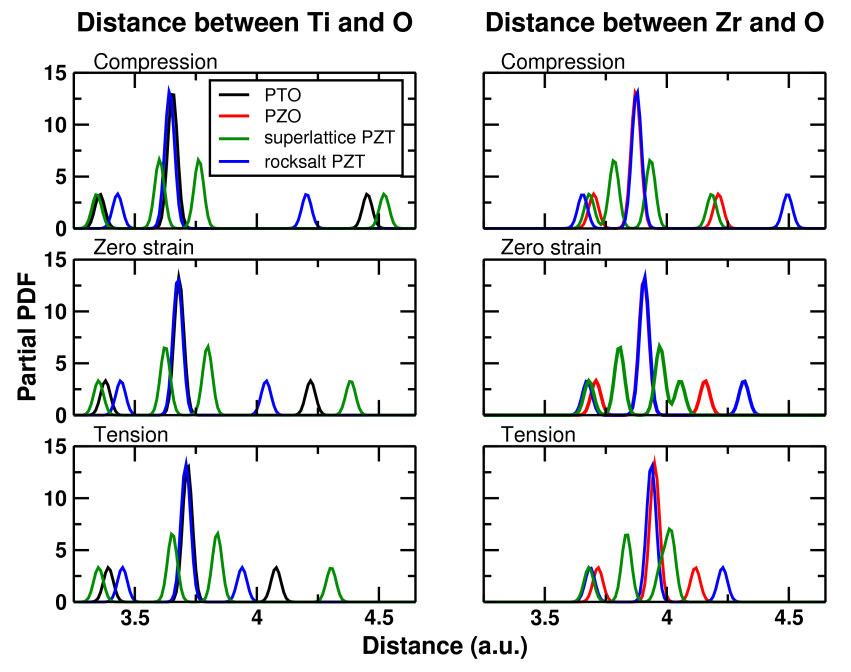

FIG. 8: Partial PDFs for Ti-O (left) and $\mathrm{Zr}-\mathrm{O}$ (right) distances. Top, middle, and lower panels show applied epitaxial strains of $\epsilon=-1.2 \%$ (compressive), 0 , and $+1.2 \%$ (tensile), respectively.

linearly using the data in Fig. 3(a), we expect contributions from the PZO and PTO components to be 0.62 and $0.77 \mathrm{C} / \mathrm{m}^{2}$. This averages to $0.70 \mathrm{C} / \mathrm{m}^{2}$, which is again very close to the calculated value of $0.72 \mathrm{C} / \mathrm{m}^{2}$.

\section{Local distortions and partial distribution functions}

The local strain arguments above suggest that in the superlattice the $\mathrm{TiO}_{2}$ layer contributes more to the net polarization than the $\mathrm{ZrO}_{2}$ layer, whereas in the rock-salt structure the $\mathrm{Ti}$ and $\mathrm{Zr}$ cells make approximately equal contributions. The contributions of the $\mathrm{Ti}$ and $\mathrm{Zr}$ ions are roughly proportional to their displacements relative to the surrounding oxygen octahedra.$^{27,28}$ The calculated relative displacements are presented as partial pair distribution functions (PDFs) ${ }^{29}$ for $\mathrm{Ti}-\mathrm{O}$ and $\mathrm{Zr}-\mathrm{O}$ neighbors in Fig. 8 The first peak at the shortest distance in each of the distributions corresponds to the $\mathrm{Ti}$ or $\mathrm{Zr}$ bonded to the $\mathrm{O}$ atom located in the nearest $x y$-oriented (basal) $\mathrm{PbO}$ plane. This $\mathrm{Zr}-\mathrm{O}$ bond length is almost identical in the two PZT structures, while the $\mathrm{Ti}-\mathrm{O}$ bond length is about $2.5 \%$ shorter in the superlattice than the rock-salt structure. This supports the hypothesis that in the superlattice structure the $\mathrm{TiO}_{2}$ layer contributes more to the net polarization than does the $\mathrm{ZrO}_{2}$ layer. In addition, the $\mathrm{Ti}-\mathrm{O}$ bond is shorter in the superlattice and the spacing between $\mathrm{PbO}$ layers is smaller, leading to a larger energy barrier in the superlattice as compared to the rock-salt structure.

The sensitivity of a crystal's polarization to its strain can also be related to the atomic structure. From Fig. 3 it is clear that PTO is more sensitive to applied strain than is the case for PZT or PZO. The PDFs indicate
Distance between $\mathrm{Pb}$ and $\mathrm{O}$

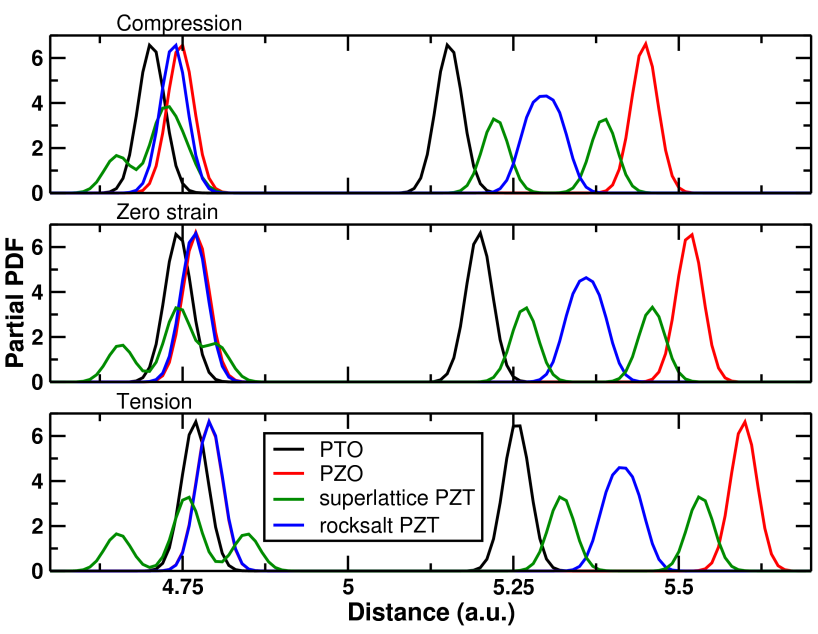

FIG. 9: Partial PDF of $\mathrm{Pb}-\mathrm{O}$ distances. Top, middle, and lower panels show applied epitaxial strains of $\epsilon=-1.2 \%, 0$, and $+1.2 \%$, respectively.

that the change in Ti-O bond length under applied load in PTO is larger than in PZT, and larger than that of Zr$\mathrm{O}$ bonds in PZT or PZO. It is this large change in bond length that leads to the change in PTO polarization with applied strain.

A partial $\mathrm{PDF}$ of $\mathrm{Pb}-\mathrm{O}$ separations is shown Fig. 9 , The shorter distances (those below 5 a.u.) correspond to $\mathrm{Pb}$ and $\mathrm{O}$ atoms lying in the same $x z$ or $y z$ plane. The superlattice has enough flexibility that the shortest of these can remain almost independent of strain, while the others show a modest shift in bond length with strain. On the other hand, the longer bonds in the right part of the figure, which correspond to $\mathrm{Pb}-\mathrm{O}$ bonds in the basal plane, show much larger shifts because they have little choice but to scale linearly with the $a$ lattice parameter. In rock-salt PZT the structure has so little flexibility that its $\mathrm{Pb}-\mathrm{O}$ bond distances are essentially just averages of the PTO and PZO ones, as can be seen in Fig. 9. In the PZT superlattice, on the other hand, the peak splits strongly because there are two $\mathrm{PbO}$ interplanar distances, with the peaks near 5.45 and 5.25 a.u. corresponding to $\mathrm{Pb}$ bonds with $\mathrm{O}$ atoms in a $\mathrm{TiO}_{2}$ or $\mathrm{ZrO}_{2}$ plane, respectively. Understanding the spatial relationships between the $\mathrm{Pb}$ and the basal $\mathrm{O}$ atoms helps to further illuminate the nature of the partial PDFs for $\mathrm{Ti}-\mathrm{O}$ and $\mathrm{Zr}-\mathrm{O}$ shown in Fig. 8 .

The partial PDFs in Fig. 10 show the Ti-Pb and $\mathrm{Zr}-$ $\mathrm{Pb}$ separations. The distributions look very reminiscent of those shown for $\mathrm{Pb}-\mathrm{O}$ distances in the right part of Fig. 9, except that here there is almost no shift with strain. The peak associated with the rock-salt structure splits because the ferroelectric distortions of the Ti and $\mathrm{Zr}$ atoms differ, but the splitting is quite small $(<0.01$ a.u.). The $\mathrm{Pb}$ and $\mathrm{Ti}$ or $\mathrm{Zr}$ atoms are not bonded to one another, but interact indirectly through shared $\mathrm{O}$ atoms. 


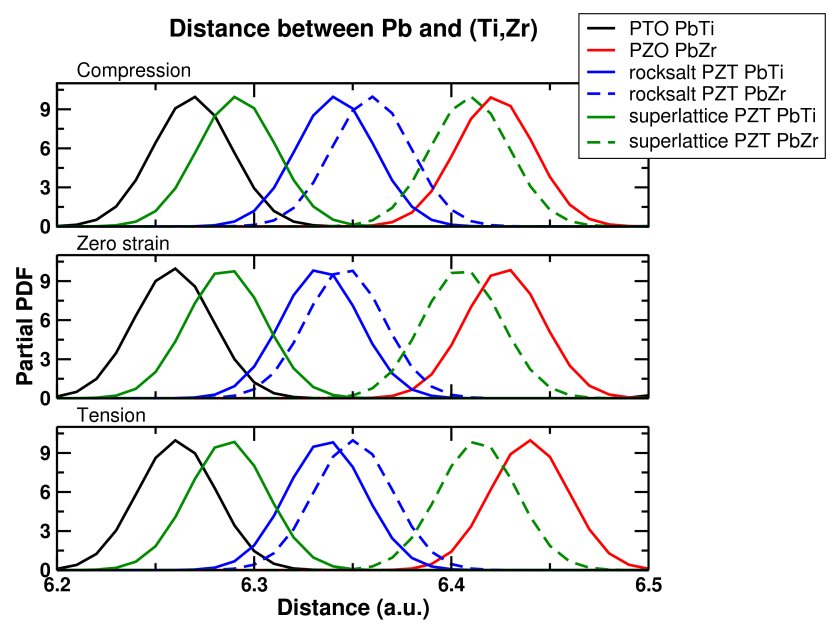

FIG. 10: Partial PDF of $\mathrm{Pb}-\mathrm{Ti}$ and $\mathrm{Pb}-\mathrm{Zr}$ distances. Top, middle, and lower panels show applied epitaxial strains of $\epsilon=-1.2 \%, 0$, and $+1.2 \%$, respectively.

\section{B. Domain-wall properties}

We turn our attention now to the results on domain walls presented in Sec. IIIC. We have inspected the atomistic structure of the domain walls, and find that in all cases they are only a few atomic planes thick. To illustrate this, the polarization profiles atomic displacements and obtained as one scans across the domain wall are plotted in Figs. 11, 12 for both PTO and PZO at zero epi-

PTO

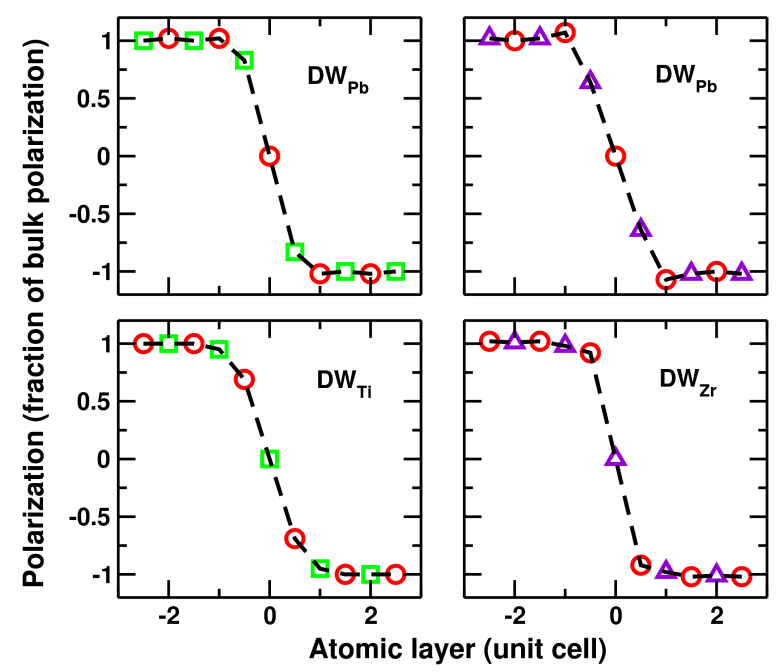

FIG. 11: Decomposition of polarization on a plane-by-plane basis across domain wall. Left, PTO; right, PZO. Top, Pbcentered walls; bottom, Ti- or Zr-centered walls. Symbols denote $\mathrm{PbO}$ (circles), $\mathrm{TiO}_{2}$ (squares), and $\mathrm{ZrO}_{2}$ (triangles) planes. taxial strain. The polarizations in Fig. 11 are determined by the procedure outlined in Ref. 7 using the atomic displacements in Fig. 12 and are normalized such that the bulk polarization has a value of 1 . Similar domain-wall thicknesses are observed for PZT. These results confirm those obtained for PTO in Ref.7, and it is clear that the $180^{\circ}$ domain walls are atomistically sharp, at least at zero temperature. Since the domain walls are all about equally narrow, it seems likely that their energies should correlate mainly with the energy cost of introducing an atomic plane in which the ferroelectric distortions are absent. This hypothesis is consistent with the observed increase of domain-wall energy with compressive strain, which makes the bulk more ferroelectric, thus introducing a larger penalty for a layer that is not ferroelectric.

However, the barrier to domain-wall motion does not depend on the absolute energies of the domain walls, but on their relative energies, as sketched in Fig. 7(c). Consider first the energy barriers in PTO and PZO, plotted in Fig. 6. The energy barrier in PTO increases substantially moving from tensile to compressive strain. This is because the domain wall centered on the $\mathrm{TiO}_{2}$ layer is highly sensitive to strain compared to the $\mathrm{PbO}$ layer. Moving from $\epsilon=+1.2 \%$ to $-1.2 \%$ the energy of the $\mathrm{TiO}_{2}$ centered wall increases by $155 \mathrm{~mJ} / \mathrm{m}^{2}$. This is almost twice the increase of the $\mathrm{PbO}$ centered wall, which increases $81 \mathrm{~mJ} / \mathrm{m}^{2}$. The barrier to domain-wall motion is related to the difference in the domain-wall energies, and the rate of change of barrier height with applied strain is related to the difference in the rate of change of domain wall energies.

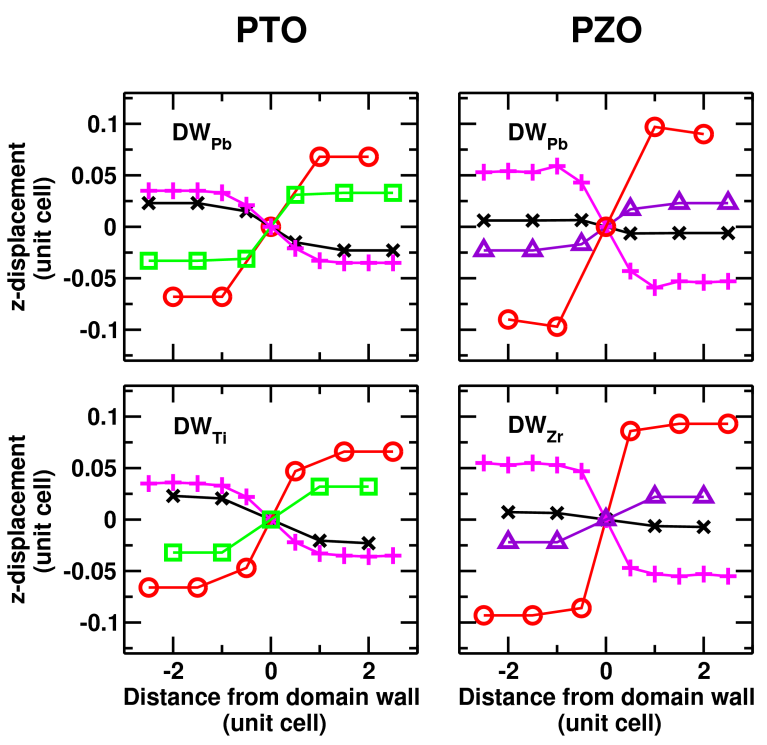

FIG. 12: Atomic displacements across domain walls. Left, PTO; right, PZO. Top, Pb-centered walls; bottom, Ti- or Zrcentered walls. Symbols denote $\mathrm{Pb}$ (circles), $\mathrm{Ti}$ (squares), $\mathrm{Zr}$ (triangles), $\mathrm{O}$ in the $x z$ or $y z$ plane (pluses), and $\mathrm{O}$ in the $x y$ plane (crosses). 
Domain wall energies in PTO, PZO, and PZT

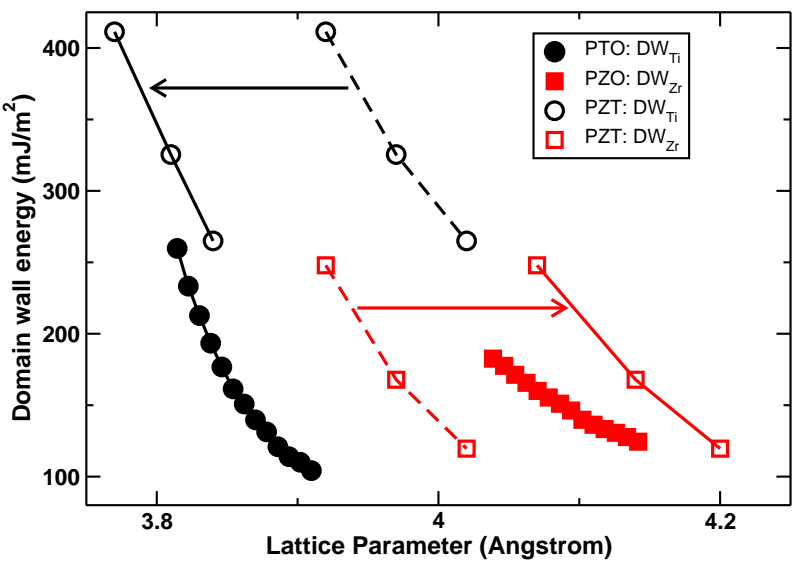

FIG. 13: Dependence of domain-wall energies on in-plane lattice constant. Solid symbols: PTO and PZO. Open symbols: PZT, plotted versus average lattice constant (connected by dashed lines) or versus local lattice constant (connected by solid lines).

In comparison, consider the energy barrier in PZO. Under compression the barrier to domain-wall motion is small because the domain walls centered on the $\mathrm{ZrO}_{2}$ and $\mathrm{PbO}$ planes have around the same energy. The barrier increases with applied tensile strain because the domain wall energies at the two planes diverge from one another in the tensile regime. Although the domain-wall energy normally increases with applied compressive load, the barrier to wall motion can decrease under some circumstances, because the barrier is a relative measure.

The sensitivity of the $\mathrm{TiO}_{2}$ layer to strain also appears to be responsible for the large barrier in the PZT superlattice. This is observed by replotting Figs. 6 and 7 in terms of the lattice parameter, as shown in Fig. 13. When the PZT domain-wall energies are plotted versus the average lattice parameter (solid symbols and open symbols connected by dashed lines), no obvious relationship emerges. On the other hand, the data clusters well when the domain-wall energies are plotted versus the $l o-$ cal lattice constant (solid symbols and open symbols connected by solid lines), defined in terms of the local lattice spacing of the $\mathrm{PbO}$ planes in the (100) direction. We can thus understand that the small spacing of the $\mathrm{TiO}_{2}$ planes is responsible for the large domain-wall energy and the large energy barrier for domain-wall motion.

\section{CONCLUSIONS}

Working with the prototypical PTO/PZO system, we have shown that the application of compressive epitaxial strain to a tetragonal ferroelectric perovskite increases its $c / a$ aspect ratio, polarization, barrier to coherent polarization reversal, ideal coercive field, and domain-wall energies. These relationships have been quantified and related to the enhancement of the atomic ferroelectric distortions and the strengthened $\mathrm{Ti}-\mathrm{O}$ bonding. The bond between the $\mathrm{Ti}$ atom and the basal $\mathrm{O}$ is highly sensitive to applied strain, as observed in the calculated partial PDFs. This sensitivity is manifested in the ordered phases of PZT, enhancing both the polarization and barrier to domain-wall motion.

In alloys, issues of local composition and strain have been shown to play an important role. Although some bulk properties, such as the average lattice parameter, obey Vegard's law, many others do not. In PZT the bulk polarization can be understood as an average of polarization contributions from each 5-atom cell, in which this contribution depends crucially both on its identity ( $\mathrm{Ti}$ or $\mathrm{Zr}$ ) and on the local strain. Similarly, we have shown that the energy of domain walls can also be understood in terms of such local effects, specifically the composition of the plane on which the domain wall is centered, and the local in-plane lattice constant for cells surrounding that plane. These results demonstrate how apparently complex properties of ferroelectric alloys can be understood as averages over single-cell properties under appropriate conditions of strain and composition.

\section{ACKNOWLEDGMENTS}

This work was supported by ONR Grant N00014-05-10054 and by the Intel Corporation via SRC Grant 2007VJ-1670. Computational resources were provided in part by the National Energy Research Scientific Computing Center (NERSC).
* Electronic address: sbeckman@iastate.edu Present address:Department of Materials Science and Engineering, Iowa State University, Ames, IA 50011-2300.

1 A.-B. Posadas, M. Lippmaa, F. J. Walker, M. Dawber, C. H. Ahn, and J.-M. Triscone, in Physics of Ferroelectrics: A Modern Perspective, edited by K. Rabe, C. H. Ahn, and J.-M. Triscone (SpringerVerlag, 2007).

${ }^{2}$ M. Dawber, K. M. Rabe, and J. F. Scott, Rev. Mod. Phys.
77, 1083 (2005).

3 J. F. Scott, Science 315, 954 (2007).

4 Y. Arimoto and H. Ishiwara, MRS Bull. 29, 823 (2004).

${ }^{5}$ Y. Kato, T. Yamada, and Y. Shimada, IEEE Trans. Electron Devices 52, 2616 (2005).

${ }^{6}$ Y.-H. Shin, I. Grinberg, I.-W. Chen, and A. M. Rappe, Nature 449, 881 (2007).

7 B. Meyer and D. Vanderbilt, Phys. Rev. B 65, 104111 (2002). 
8 N. A. Pertsev, A. G. Zembilgotov, and A. K. Tagantsev, Phys. Rev. Lett. 80, 1988 (1998).

9 O. Dieguez, K. M. Rabe, and D. Vanderbilt, Phys. Rev. B 72, 144101 (2005).

10 R. D. King-Smith and D. Vanderbilt, Phys. Rev. B 47, 1651 (1993).

11 C. Bungaro and K. M. Rabe, Phys. Rev. B 69, 184101 (2004).

12 M. Dawber, P. Chandra, P. B. Littlewood, and J. F. Scott, J. Phys.: Condens. Matter 15, L393 (2003), ISSN 09538984.

13 J. Junquera and P. Ghosez, Nature 422, 506 (2003).

14 C. Yoshida, A. Yoshida, and H. Tamura, Appl. Phys. Lett. 75, 1449 (1999).

15 S. P. Beckman and D. Vanderbilt, unpublished data (2008).

16 L. Bellaiche, A. Garcia, and D. Vanderbilt, Phys. Rev. Lett. 84, 5427 (2000).

17 I. Grinberg, V. R. Cooper, and A. M. Rappe, Phys. Rev. B 69, 144118 (2004).

18 W. Kohn and L. J. Sham, Phys. Rev. 140, A1133 (1965).

19 D. M. Ceperley and B. J. Alder, Phys. Rev. Lett. 45, 566 (1980).

$20 \mathrm{P}$.

Giannozzi

and

et

al. http://www .quantum-espresso.org

21 D. Vanderbilt, Phys. Rev. B 41, 7892 (1990).

22 The parameterization used for these pseudopotentials is available from http://www.physics.rutgers.edu/ dhv/uspp/.

${ }^{23}$ H. J. Monkhorst and J. D. Pack, Phys. Rev. B 13, 5188 (1976).

24 F. Jona and G. Shirane, Ferroelectric Crystals (Dover, 1993).

25 G. Shirane, R. Pepinsky, and B. C. Frazer, Acta Crystallographica 9, 131 (1956).

26 P. Chandra and P. B. Littlewood, in Physics of Ferroelectrics: A Modern Perspective, edited by K. Rabe, C. H. Ahn, and J.-M. Triscone (SpringerVerlag, 2007).

27 The polarization is proportional to the displacement multiplied by the Born effective charge. The Born effective charge for $\mathrm{Z}^{*}(\mathrm{Zr})$ in $\mathrm{PZO}$ is around 6 and $\mathrm{Z}^{*}(\mathrm{Ti})$ in $\mathrm{PTO}$ is around 7 , which is not a substantial difference ${ }^{28}$

${ }^{28}$ W. Zhong, R. D. King-Smith, and D. Vanderbilt, Phys. Rev. Lett. 72, 3618 (1994).

29 The partial PDFs are a histograms of atomic separations, plotted with a Gaussian smearing having a variance of 0.02 a.u. 